\title{
Special Section Guest Editorial: Satellite Hyperspectral Remote Sensing: Algorithms and Applications
}

\author{
Kun Tan, ${ }^{a, b, c}$ Xiuping Jia, ${ }^{d}$ and Antonio Plaza ${ }^{e}$ \\ ${ }^{a}$ East China Normal University, Key Laboratory of Geographic Information Science \\ (Ministry of Education), Shanghai, China \\ ${ }^{\mathrm{b}}$ East China Normal University, Ministry of Natural Resources, Key Laboratory \\ of Spatial-temporal Big Data Analysis and Application of Natural Resources \\ in Megacities, Shanghai, China \\ ${ }^{c}$ East China Normal University, School of Geographic Sciences, Shanghai, China \\ ${ }^{\mathrm{d}}$ University of New South Wales, School of Engineering and Information Technology, \\ Canberra, Australian Capital Territory, Australia \\ ${ }^{e}$ University of Extremadura, Escuela Politecnica, Hyperspectral Computing Laboratory, \\ Department of Technology of Computers and Communications, Cáceres, Spain
}

Hyperspectral images, which contain both spectral and spatial information of the scene, are an important data source in remote sensing applications. In recent years, the development of advanced computing techniques such as artificial intelligence, deep learning, and weakly supervised learning has further expanded the scope of satellite hyperspectral remote sensing.

This special section focuses on the latest research findings in satellite hyperspectral image analysis and applications, encompassing topics such as hyperspectral unmixing, change detection, precision classification, and ecological monitoring and assessment, etc.

Zhang et al. introduced the low-rank constraint and a reweighting strategy in hyperspectral unmixing, and verified the effectiveness of the proposed algorithm through simulated and real data experiments. To reconstruct hyperspectral images with higher spatial resolution, Yu et al. explored the fusion of the hyperspectral image with panchromatic and multispectral images captured by the sensors onboard ZY1-02D, and the fused hyperspectral images improve the performance in ground object classification. Chou et al. proposed a novel semisupervised dimensionality reduction method via the use of valid unlabeled samples and spatial neighborhood information in a semi-supervised graph embedding approach. A novel self-training classification algorithm that combines KNN and SVM was proposed by Ge et al., where a mixture of spectral distance, spatial distance, and local outlier factor are used to assess the sample similarity. The algorithm achieves excellent performance in several hyperspectral datasets. Deep neural networks play an important role in the field of intelligent interpretation of remote sensing data. $\mathrm{Yu}$ et al. proposed a 3D convolutional autoencoder algorithm and successfully achieved large-scale lithological mapping with the ZY1-02D hyperspectral imagery. Singh et al. combined deep convolutional neural networks and Markov random field to obtain smoother classification maps in satellite hyperspectral imagery. Wang et al. introduced a pyramidal self-attentive mechanism in deep neural networks for change detection and validated using the hyperspectral change detection dataset from ZY1.

The unique spectral information of hyperspectral imagery can represent detailed features and provide a basis for ecological monitoring and assessment. Yang et al. proposed a decision tree water index for water extraction from hyperspectral imagery, which overcomes the shortcomings of traditional multispectral water indices directly when it is applied to hyperspectral images. Wang et al. used a dimension reduction method based on the variable importance projection (VIP) and segmented principal component analysis (SPCA) method for the water quality parameters retrieval from GF5 satellite hyperspectral imagery. Ghosh et al. used multi-temporal hyperspectral imagery to obtain abundance maps through the spectral unmixing method and used the Markov chain model to analyze the dynamics of change and predict future scenarios. Shang et al. explored the application potential of ZY1-02D satellite hyperspectral data in estimating soil $\mathrm{Cu}$

(C) 2021 Society of Photo-Optical Instrumentation Engineers (SPIE) 
content in mining areas. Wu et al. assessed the soil heavy metal pollution based on the estimated maps and investigated the scale effect between airborne and satellite hyperspectral imagery.

These unique studies contributed to the development of satellite hyperspectral technology and laid the foundation for hyperspectral applications in a variety of fields.

The authors, the reviewers, and the Journal of Applied Remote Sensing production team have contributed greatly to the launch of this special section. The guest editors of this special section would like to thank everyone for their hard work. 